SCIENTIFIC LETTER

\title{
Is there a sex bias in implantable cardioverter-defibrillator referral and prescription?
}

\author{
A D Staniforth, S C Sporton, N M Robinson, J Cooper, M J Earley, A W Nathan, R J Schilling
}

Heart 2004;90:937-938. doi: 10.1136/hrt.2003.013136

$\mathrm{T}$ he trandolapril cardiac evaluation (TRACE) investigators reported a male to female ratio of sudden cardiac death (SCD) in coronary disease of $1.4: 1 .^{1}$ This confirmed that women with coronary artery disease were at a higher risk of SCD than the Framingham investigators had suggested. ${ }^{2}$ The low proportion of female subjects undergoing implantable cardioverter-defibrillator (ICD) implantation is well recognised and these data lead us to question whether there could be a sex bias in the referral or prescription of ICDs in patients with coronary artery disease.

\section{METHODS}

We analysed the characteristics of 514 ICD implants performed between January 1990 and October 2002. Early ICD recipients were largely implanted for secondary prevention following aborted SCD. Subsequent implant practice evolved in line with the results of large clinical trials (AVID (anti-arrhythmics versus implantable defibrillators) and MADIT-1 (multicenter automated defibrillator implantation trial)). There was no upper age limit for ICD implantation. Demographic information contained in our ICD database was entered prospectively at the time of implant. All subjects underwent coronary angiography. Ejection fraction was estimated by either echocardiography or left ventricular angiography. Patients were reviewed at ICD clinic visits and therapies were documented as appropriate, inappropriate, or electrical storm following review of stored electrograms. Before the advent of generator electrogram storage, this distinction was made on the basis of the presence of antecedent symptoms relieved by therapy. We hypothesised that if we were discriminating against females, we might expect to observe differences between male and female subjects in their risk factors for arrhythmia or their subsequent arrhythmic events and survival.

Numerical data were analysed using the Student's $t$ and Mann-Whitney U tests. Group comparisons of categorical data were evaluated using the $\chi^{2}$ test. Survival curves were calculated according to the Kaplan-Meier actuarial method.
Differences in survival rates were compared using the log rank test.

\section{RESULTS}

Five hundred and fourteen implants took place in 406 patients with a total follow up of 13904 months. Females accounted for $16 \%$ of implant subjects. Seventy four per cent of patients had coronary artery disease (CAD); 31\% (32/104) of ICD patients without CAD were female, compared with only $11 \%(34 / 302)$ with CAD $(p<0.005)$. This reflected the skewed nature of the male to female ratio of CAD. The sex distribution of implants for CAD did not change between 1990 and 2002. Only 5\% of CAD implants were for primary prevention; MADIT-2 had not influenced our implant practice by 2002 .

Male and female subjects were matched for age, ejection fraction, and arrhythmic indication for implant (table 1). Male subjects had worse coronary disease than females $(\mathrm{p}<0.005)$. The mean $($ SEM) number of coronary arteries with significant disease in males and females was $2.2(0.1)$ and 1.6 (0.2). An electrophysiology study (EPS) was performed in $66 \%$ of patients. There was no difference in ventricular tachycardia (VT)/ventricular fibrillation (VF) inducibility between males and females undergoing EPS ( $94 \%$ and $88 \%$, respectively).

Kaplan-Meier analysis showed no survival difference according to sex (male median survival 120 months, female 88 months, $\mathrm{p}=0.09$ ). Males and females were equally as likely to experience appropriate therapy (male median time to first appropriate therapy 28 months, female 18 months, $\mathrm{p}=0.34$ ) or electrical storm (the proportion of males

Abbreviations: AVID, anti-arrhythmics versus implantable defibrillators; CAD, coronary artery disease; EPS, electrophysiology study; ICD, implantable cardioverter-defibrillator; MADIT, multicenter automated defibrillator implantation trial: $S C D$, sudden cardiac death; TRACE, trandolapril cardiac evaluation; VF, ventricular fibrillation; VT, ventricular tachycardia

Table 1 Baseline characteristics of ICD patients with coronary artery disease.

\begin{tabular}{rlll}
\hline & Male $(\mathbf{n}=\mathbf{2 6 3})$ & Female $(\mathbf{n}=\mathbf{3 4})$ & $\mathrm{p}$ Value \\
\hline Age (years) & $65(1)$ & $64(2)$ & 0.56 \\
Ejection fraction (\%) & $32(1)$ & $32(2)$ & 0.96 \\
Diabetes mellitus (\%) & $32 / 243(13)$ & $6 / 30(20)$ & 0.31 \\
Hypertension (\%) & $57 / 239(24)$ & $9 / 30(30)$ & 0.68 \\
Number of diseased arteries & $2.2(0.1)$ & $1.6(0.2)$ & $<0.005$ \\
Index arrhythmia $\quad$ VT $(\%)$ & $180 / 259(70)$ & $25 / 34(74)$ & \\
VF $(\%)$ & $65 / 259(25)$ & $8 / 34(74)$ & 0.81 \\
PP (\%) & $14 / 259(5)$ & $1 / 34(3)$ & \\
Inducible at EPS (\%) & $159 / 169(94)$ & $23 / 26(88)$ & 0.25
\end{tabular}

Age, ejection fraction, and number of diseased arteries at coronary angiography are presented as mean (SEM) EPS, electrophysiology study; PP, primary prevention; VF, ventricular fibrillation; VT, ventricular tachycardia. 
without electrical storm at 12 and 48 months was $76 \%$ and $60 \%$, respectively, females $78 \%$ and $71 \%$, respectively; $\mathrm{p}=0.57)$.

\section{DISCUSSION}

We report a large series of consecutive patients from a single centre. The proportion of female implants matches that of the UK $(18 \%)^{3}$ and Danish registries $(20 \%),{ }^{4}$ and the results of published clinical trials (AVID, MADIT-1, and MADIT-2). In subjects with $\mathrm{CAD}$, we observed an implant ratio of $8: 1$ male excess. Framingham originally suggested that the risk of SCD in CAD was a 4:1 male excess. ${ }^{2}$ The TRACE investigators recently reported a mere 1.4:1 male loading. ${ }^{1}$ It is concerning that females continue to be under represented in ICD implant activity both from national databases and published trial data.

If females were finding it harder to prove themselves worthy of an ICD at our institution, then we might expect them to have greater baseline markers of arrhythmic risk, more arrhythmia, and poorer survival. Our results showed that male and female subjects were equally matched for baseline co-morbidity, arrhythmia presentation, and VT/VF inducibility at EPS. Females were just as likely to experience appropriate therapy, electrical storm, or death as males on follow up. We concluded that there was no evidence of frank, systematic in-house discrimination against females at ICD prescription.

Sex bias might not be the only explanation for differences in implant practice between males and females. An alternative interpretation might be that female subjects with CAD present at an older age and with more co-morbidity and that it was these subjects who were not receiving an ICD. It was a limitation of our study that we have no data concerning patients assessed for an ICD who were not implanted. The National Health Service has never imposed an upper age limit for ICD implantation. Furthermore, the only co-morbidity that would restrict ICD implantation in our institution is one expected to shorten survival to less than six months.

We were suspicious that the "missing" women were not reaching us from our referring centres. There could be at least four explanations for this: the women were erroneously perceived to be at low risk of SCD; they were not being referred because of increased age and greater co-morbidity; older subjects with co-morbidity were themselves declining to be referred; or they were already dead (women have poorer survival rates following myocardial infarction and SCD). All four explanations are compatible with the observation that our females had less severe CAD. We cannot confirm which explanation is correct because we have no data concerning patients who did not reach our centre. Further studies are required to identify why women are less likely to receive an ICD than men. We suggest that these should be directed towards examining the different outcomes in males and females at risk of arrhythmia before they reach hospital.

We conclude that our data indicate that too few females with CAD are referred for an ICD. The reason for this is unclear.

\section{ACKNOWLEDGEMENTS}

Dr AD Staniforth is supported by an educational grant from Guidant Ltd.

\section{Authors' affiliations}

A D Staniforth, N M Robinson, M J Earley, A W Nathan, R J Schilling, Department of Cardiology, St Bartholomew's Hospital, London, UK S C Sporton, The Heart Hospital, University College London Hospitals NHS Trust, London, UK

J Cooper, Centre for Cardiovascular Genetics, Department of Medicine, University College London, London, UK
Correspondence to: Dr Andrew D Staniforth, St Bartholomew's Hospital, Dominion House, 60 Bartholomew Close, London EClA 7BE, UK; adstaniforth@beeb.net

Accepted 25 September 2003

\section{REFERENCES}

1 Abildstrom SZ, Rask-Madsen C, Ottesen MM, et al. Impact of age and sex on sudden cardiovascular death following myocardial infarction. Heart 2002;88:573-8.

2 Kannel WB, Wilson PW, D'Agostino RB, et al. Sudden coronary death in women. Am Heart J 1998;136:205-12.

3 Cunningham D. UK pacemaker and ICD database 1998-2000. www.ccad3.biomed.gla.ac.uk/bpeg.

4 Moller M, Arnsbo P. Danish pacemaker and ICD register 2001. www.pacemaker.dk/Contact.html.

\section{WEB TOP 10}

www.heartinl.com

These articles scored the most hits on Heart's website during May 2004

1 Management of acute coronary syndromes: an update KAA Fox

June 2004;90:698-706. (Education in Heart)

2 Echocardiography in infective endocarditis

A Evangelista, MT Gonzalez-Alujas

June 2004;90:614-7. (Mini-symposium)

3 Diagnostic criteria and problems in infective endocarditis $B D$ Prendergast

June 2004;90:611-13. (Mini-symposium)

4 British Cardiac Society Working Group on the definition of myocardial infarction

KAA Fox, J Birkhead, R Wilcox, C Knight, J Barth June 2004;90:603-9.

5 Assessing the risk of sudden cardiac death in a patient with hypertrophic cardiomyopathy

MP Frenneaux

May 2004;90:570-5. (Education in Heart)

6 Free radicals and redox signalling in cardiovascular disease: introduction

AM Shah, KM Channon

May 2004;90:485. (Mini-symposium)

7 The pathophysiology of myocardial ischaemia

DC Crossman

May 2004;90:576-80. (Education in Heart)

8 Indications and optimal timing for surgery in infective endocarditis

F Delahaye, M Celard, O Roth, G de Gevigney

June 2004;90:618-20. (Mini-symposium)

9 Infective endocarditis: introduction

$P$ Tornos

June 2004;90:610. (Mini-symposium)

10 Utility of echocardiography in the evaluation of individuals with cardiomyopathy

MJ Wood, MH Picard

June 2004;90:707-12. (Education in Heart)

Visit the Heart website for hyperlinks to these articles, by clicking on "Top 10 papers"

www.heartinl.com 